\title{
Les techniques modernes amé- liorent la prise en charge du diabète
}

\section{Roger Lehmann}

Prof., responsable de la diabétologie, clinique d'endocrinologie, diabète et alimentation clinique, Hôpital universitaire de Zurich

Les derniers développements dans le domaine des capteurs de glucose et des pompes à insuline induiront à court terme une augmentation du coût de traitement du diabète de type 1 . En contrepartie, ces progrès vont considérablement améliorer la qualité de vie des patients et le bon contrôle à long terme de la glycémie, et permettre d'éviter des complications, d'où d'importantes économies dans le secteur de la santé.

Quelque 25000 personnes présentent en Suisse un diabète de type 1 , dont les cellules productrices d'insuline du pancréas sont détruites. Ce manque d'insuline empêche le transport du glucose du sang vers les cellules du corps, et le taux de glycémie augmente. Cela entraîne non seulement les symptômes typiques du diabète, mais également des maladies associées telles que les maladies cardiovasculaires, une baisse de la vision jusqu'à la cécité, une insuffisance rénale ou des atteintes neurologiques.

Pour les patients concernés, l'apport d'insuline et la bonne régulation du taux de glycémie sont un défi permanent. En tenant compte de leur alimentation et de leur activité, ils doivent adapter leur dose d'insuline sur la base de leur taux de sucre actuel, afin d'éviter une hypoglycémie ou un manque d'insuline qui pourraient leur être fatal.

\section{Les progrès du traitement du diabète dans la vie d'un patient octogénaire}

Le cas d'un octogénaire dynamique, présentant un diabète de type 1 depuis l'âge de 7 mois, illustre parfaitement les progrès réalisés dans la manière de contrôler cette maladie. A sa naissance en 1937 - 16 ans après la découverte de l'insuline et 14 ans après sa mise sur le marché - il n'existait que l'insuline d'action rapide qui devait être injectée 3 à 4 fois par jour à l'aide d'une seringue en verre dont l'aiguille en acier devait être désinfectée à l'alcool et affutée, au besoin, à l'aide d'une lime à métaux. Pour déterminer le taux de sucre, on portait l'urine à ébullition sur un bec Bunsen selon le Test de Benedict; ce n'est qu'en 1941 que le comprimé Clinitest a permis la détection de sucre à l'aide de quel- ques gouttes d'urine et d'eau. Ce patient a dû attendre plus de 30 ans pour mesurer son taux de glycémie, avec le développement dans les années 70 de l'hémoglucotest. Les premiers lecteurs de glycémie numériques sont arrivés sur le marché en 1980. Ce patient a également été témoin de nombreuses étapes dans l'évolution de l'insuline: de l'insuline basale d'action lente introduite en 1946 (Insulatard) aux insulines humaines fabriquées par génie génétique, puis aux insulines analogues.

\section{La mesure du glucose en continu évite l'hypoglycémie}

Après avoir failli mourir d'une hypoglycémie sévère, le patient demande un système de mesure du glucose en continu avec alerte en cas de baisse de sucre. Les systèmes de mesure du glucose en continu (continuous glucose monitoring CGM) sont utilisés dans le traitement du diabète depuis 1999. Il préfère le système CGM qui, en plus de cette fonction d'alerte, alarme également ses personnes de contact via smartphone. Il reçoit une formation personnalisée à cette technologie par l'équipe du diabète, afin de se familiariser avec les valeurs du système (taux de glucose et indications de tendance) et de savoir quelles réponses sont adéquates.

\section{Détection d'un diabète de type 1 chez une jeune femme}

Lors d'un séjour à l'étranger, un diabète est diagnostiqué chez une patiente de 21 ans et traité à l'insuline lente (avant le coucher) et à l'insuline rapide avant les repas (basal-bolus). Ces traitements actuels, comparé à 
celui de 1937, ne sauraient être plus différents. Même si l'apprentissage des éléments de base comme la technique d'injection, la mesure du taux de glycémie et surtout l'identification et l'estimation des hydrates de carbone, est resté le même, l'utilisation actuelle d'insuline analogue ultra-rapide et d'insuline basale à action ultra-lente permet d'obtenir un bien meilleur profil de l'insuline et de la glycémie. Depuis juillet 2017, les caisses-maladie prennent en charge pour tous les patients avec une telle insulinothérapie, un système Flash de monitoring du glucose (Flash glucose monitoring, FGM), une nouvelle méthode de mesure du taux de glucose. Dès la première consultation, la patiente a bénéficié de ce système qui consiste en un capteur que l'on pique sous la peau; au bout d'une heure, elle peut mesurer son taux de glucose dans les tissus pendant 14 jours, aussi souvent qu'elle le souhaite. Elle peut ainsi connaître après chaque repas si le rapport insuline/glucides est équilibré ou si elle doit modifier un paramètre. Elle n'a donc plus besoin de se piquer le doigt 4 à 6 fois par jour pour mesurer son taux de glycémie. Avec ce nouveau système, la plupart des patients mesurent leur taux de sucre environ 10 à 16 fois par jour et le graphique indique l'évolution sur les 8 dernières heures.

\section{Sur la voie d'un «pancréas artificiel»}

Ces systèmes simples de monitoring du glucose ne détectant pas en avance une éventuelle hypoglycémie, ce qui serait essentiel la nuit notamment, la jeune patiente devra tôt ou tard se demander si un capteur de glucose combiné à une pompe à insuline ne permettrait pas d'améliorer encore plus l'équilibration de la glycémie. Avec un tel système, le capteur de glycémie communique directement avec la pompe à insuline qui peut interrompre l'apport d'insuline avant l'apparition d'une hypoglycémie. Si le taux de sucre remonte, l'apport d'insuline reprend. La dernière version de cette pompe à insuline avec capteur de glycémie (670 G), qui devrait être mise sur le marché en Suisse l'année prochaine, prévient non seulement des hypoglycémies, mais corrige aussi automatiquement les taux de glucose élevés. Elle fonctionne ainsi comme un pancréas artificiel, mais semi-automatique. En effet, la quantité d'insuline doit encore être déterminée pour un repas donné et la quantité de glucides estimée correctement. Après la saisie du glucose et de la quantité de glucides, la quantité d'insuline nécessaire est calculée sur la base de règles individuelles. Au vu de ces avancées, l'un des objectifs technologiques du traitement du diabète - soit le développement d'un pancréas artificiel capable de contrôler automatiquement et de manière autonome l'apport d'insuline et le glucose - ne semble plus très éloigné.

\section{Des économies malgré des coûts initiaux plus élevés}

L'utilisation de moyens auxiliaires numériques améliore la qualité de vie de ces patients chroniques en leur simplifiant l'équilibration de la glycémie, mais rend le traitement actuel plus onéreux. C'est pourquoi les

\section{Eviter les hypo- ou hyperglycémies réduit considérablement les hospitalisations et les complications.}

systèmes CGM ne sont remboursés que si une amélioration est confirmée au bout de 6 mois. Ces systèmes étant relativement nouveaux, il n'existe pas encore d'études prospectives de grande ampleur sur leur rentabilité; on sait toutefois que l'évitement d'hypo- ou d'hyperglycémies réduit considérablement les hospitalisations et les complications. Ces économies devraient compenser les coûts initiaux élevés, en plus du fait que la qualité de vie des diabétiques et leur productivité professionnelle devraient nettement s'accroître.

\section{Résumé}

Les options de traitement pour les diabétiques de type 1 se sont considérablement améliorées depuis la découverte de l'insuline, mais aussi au cours des dernières années. De nouveaux systèmes de mesure de la glycémie et d'apport d'insuline permettent un monitoring continu et moins éprouvant du glucose ainsi qu'une régulation optimale de l'insuline. Ils ont des répercussions positives sur le quotidien et la qualité de vie des patients, et permettent d'éviter des maladies associées, des urgences ou des complications onéreuses. 